\title{
Mucosal abnormalities at the anastomosis site in patients who have had intestinal resection for colonic
}

\section{cancer}

\author{
JP SUNTER, MJ HIGGS, ${ }^{*}$ WK COWAN
}

From the Departments of Pathology and *Surgery, Queen Elizabeth Hospital, Gateshead, and the Department of Pathology, University of Newcastle upon Tyne

SUMMARY Twenty eight patients with colonic cancer, who were asymptomatic after intestinal resection and anastomosis, underwent colonoscopy as part of their routine follow up, and biopsies were obtained from the anastomosis and several other sites. Sections were stained by haematoxylin and eosin, several methods for mucin, and by the peroxidase-antiperoxidase method for carcinoembryonic antigen. Non-specific inflammatory changes were seen at the anastomosis in 11 of the 28 cases, apparent in several two years after operation; focal surface ulceration was seen in over half these samples. Neither dysplastic nor adenomatous change was detected, but at seven anastomoses the so called transitional change, which has been regarded as a preneoplastic change, was apparent. There was no consistent alteration in carcinoembryonic antigen reactivity.

It is concluded that there is morphological evidence of a continued stimulus to regenerative activity at some anastomoses and that this may represent a promoting factor enhancing further carcinogenesis.

Surgical resection is the only form of treatment which offers any real hope of cure in cases of colorectal cancer. More than half of all patients with the disease present at a stage when a resection designed at producing cure appears possible.' But after operation some of these patients will die of the effects of metastatic disease not apparent at resection, while others will succumb to the effects of local recurrence. These local recurrences account for the deaths of perhaps $10-15 \%$ of individuals who have had "curative" resections ${ }^{12}$ and may develop in the peritoneum or in the abdominal wound, but are particularly likely to arise at the site of the intestinal anastomosis. The development of carcinoma at the anastomosis has conventionally been regarded as representing a true recurrence of the original primary growth, due either to inadequacy of local resection in the first place or to implantation of exfoliated but viable tumour cells during the operation. There is no doubt that some anastomotic cancers must represent what is, in effect, a failure of surgical technique. There are, however, a number of reasons for considering the possibility that anastomotic cancers in fact represent second primary growths, the development of which has been promoted by some abnormality at the anastomosis.

In the first place large bowel cancer is not infrequently a multicentric disease, and both synchronous and metachronous carcinomas are not uncommon. ${ }^{34}$ It is perhaps not surprising therefore that

Accepted for publication 12 December 1984 generalised mucosal abnormalities have been described in the non-neoplastic mucosae of patients with ordinary large bowel carcinoma and that these abnormalities have been regarded as having significance as a preneoplastic state.$^{5-7}$ If this is true the promoting influences resulting from the presence of an intestinal anastomosis might result in neoplastic development in the mucosa at that site, a change which would not occur in the absence of such influences. It is noteworthy in this context that local recurrence is more common in cases of carcinoma of the rectosigmoid treated by anterior resection than in cases treated by abdominoperineal resection, regardless of the margin of clearance of the tumour. ${ }^{8}$ While part of this difference may be accounted for by differences in the extent of clearance of perirectal soft tissue, another important factor could well be the presence in the anterior resection cases of an anastomotic line.

In the animal models of colorectal carcinogenesis which involve the administration of chemical carcinogens there is a striking tendency for primary colonic neoplasms to arise at sites of intestinal anastomosis. This is true regardless of whether the construction of the anastomosis precedes ${ }^{y}$ or follows ${ }^{10}$ carcinogen treatment, suggesting that some proliferative instability induced by the presence of the anastomosis may be a critical factor determining the site at which successful neoplastic development occurs. Perhaps of even greater interest, given the exceptional rarity of spontaneous intestinal carcinomas in laboratory rodents, are observations 
relating to the development of adenocarcinomas in colostomy sites in rats not subjected to carcinogen treatment" and even occasionally in the colonic anastomoses of control animals being used in carcinogenicity studies. ${ }^{12}$

In view of these arguments it would seem probable that at least some anastomotic recurrences in human patients do constitute metachronous "suture line primaries," and this has implications as far as patient management is concerned. The investigation of patients with established carcinoma at the anastomosis site can give information of only limited value, since it is difficult to be sure on pathological grounds whether the tumour is a recurrence or a second primary even in the presence of some unusual histological feature, and such a distinction is quite impossible in the case of more ordinary growths. Several morphological abnormalities of large intestinal mucosa have been described, however, in situations associated with an enhanced risk of development of colonic cancer ${ }^{56}$ and their significance as preneoplastic phenomena implied, while the subsequent dysplasia-carcinoma sequence is now generally accepted. ${ }^{13}$ We have therefore investigated by means of colonoscopy and biopsy the anastomosis site in a group of healthy patients, who had previously had surgical resection for colonic cancer, in order to document any early mucosal changes which might imply de novo tumorigenesis.

\section{Material and methods}

The study group consisted of 28 asymptomatic individuals who were being routinely followed as outpatients after intestinal resection and immediate anastomosis for tumours of the large bowel. None of the patients suffered from inflammatory bowel disease or polyposis-in other words, their tumours had been ordinary large bowel neoplasms. In every case a "curative" resection had been performed and margins of resection had been judged adequate pathologically. As part of the routine follow up each individual was subjected once to colonoscopic examination. The colonoscopies were staggered so that observations were included from about 6 months after resection (an interval considered long enough to allow for normal mucosal healing), about 12 months after resection, and about 24 months after resection. Care was taken to ensure that the whole colon was visualised, and any areas of abnormality - for example, polyps - were biopsied; particular attention was paid to the state of the anastomosis. Multiple mucosal biopsies (usually three or four pieces) were obtained from the site of the anastomosis and also from apparently normal colonic mucosa proximal and distal to the anastomosis (in left sided resections) and distal to the anastomosis in cases where right hemicolectomy and ileocolic anastomosis had been performed.
The biopsies were fixed overnight in neutral buffered formol-saline solution and then routinely processed and embedded in paraffin wax. Multiple duplicate histological sections $4 \mu \mathrm{m}$ thick were prepared at several levels. One set was stained immediately with haematoxylin and eosin for diagnostic purposes and a histopathological assessment made. The rest of the sections were retained unstained and subsequently were batched for further staining procedures. These consisted of several stains for mucus that is, the periodic acid Schiff reaction after pretreatment with diastase, the high iron diamine/alcian blue $(\mathrm{pH} 2.5)$ technique $^{14}$ and Shikata's orcein method, ${ }^{14}$ and a peroxidase-antiperoxidase method (Dako) for carcinoembryonic antigen.

When all the histological material had been assembled the slides, including the original haematoxylin and eosin stained sections, were assessed blindly by two observers.

\section{Results}

\section{PATIENTS}

Of the 28 patients who comprised the study group $\bar{\emptyset}$ there were 15 men and 13 women. The mean age at 0 colonic resection for the men was 62 years (range 27-78) and for the women 67 years (range 38-79). In 12 cases the tumour was situated in the caecum or ascending colon and in one case the growth was in the proximal transverse colon; these individuals were treated by right hemicolectomy and thus had ileo-colic anastomoses. Four patients with tumours of the descending colon were treated by left hemicolectomy and the 11 with growths of the sigmoid colon or upper rectum by sigmoid colectomy or anterior resection; these patients therefore had 8 colonic anastomoses. In all cases the anastomoses were fashioned by hand in two layers using routinely black silk sutures and catgut. Histologically, the tumours proved unequivocally to be primary colonic tumours of epithelial origin. There were nine well 응 differentiated adenocarcinomas, 12 moderately well $>$ differentiated adenocarcinomas, and six poorly differentiated adenocarcinomas; one lesion was finally $N$ classified as a large benign tubulovillous adenoma. In all cases resection was judged adequate and the $\tilde{O}$ operations could therefore be considered "cura- $N$ tive." Synchronous tumours were seen in the resec- $\bar{\sigma}$ tion specimen, or were visualised and biopsiedo endoscopically before the resection, in five patients. In one case the synchronous tumour was a second $\stackrel{\oplus}{?}$ carcinoma, while in the other four the lesions were 0 single small tubular adenomas. A further three $\stackrel{P}{P}$ patients had coexistent metaplastic polyps.

Following resection all the patients recovered $\stackrel{\vec{Q}}{\mathrm{D}}$ uneventfully and appeared to be healthy on subsequent follow up. None had any symptoms which could possibly have been related to abnormalities ato응 the site of the anastomosis when colonoscopy was 
performed. This took place about 6 months after resection in five patients, about 12 months after resection in 12 , and about 24 months after resection in 11 .

\section{COLONOSCOPIC FINDINGS}

Colonoscopy was usually easy in these patients, especially those who had had left sided resections, and an adequate view of the colorectal mucosa and the anastomosis site was obtained in all patients.

The anastomosis was discernible as a region of attenuation of the normal pattern of mucosal folds, and in some cases a puckering of the mucosa was evident. Black silk sutures were quite often visible through mucosa which was obviously intact, but in some five cases there was clear evidence of inflammation; in two cases small polypoidal masses, apparently of granulation tissue, were seen. In only one case was there the suggestion of any tumour recurrence and this was in fact negative histologically (see below). There was no evidence of stenosis at any anastomosis.

In most patients the general colonoscopic findings were essentially negative, although so called melanosis coli was common. In five individuals single small polyps remote from the site of the anastomosis were visualised and biopsied; these proved to be benign tubular adenomas. Three more individuals had several simple metaplastic polyps.

\section{MICROSCOPICAL CHANGES AT THE}

ANASTOMOSIS

No appreciable technical difficulties in obtaining mucosal biopsies from the anastomosis site were experienced, the samples from the anastomosis being similar in size and state of preservation to those obtained from other sites in the bowel. Inspection of haematoxylin and eosin stained sections of the latter showed no evidence of any generalised mucosal abnormality in any case.

The haematoxylin and eosin preparations of the anastomoses showed that in most cases the mucosa was perfectly normal and indistinguishable from the samples obtained from other parts of the bowel. In 11 cases, however, they showed clear evidence of mucosal inflammation. In some fragments this evidence consisted merely of a slight increase in the numbers of mononuclear cells in the lamina propria (Fig. 1), but in most cases the changes were more striking. They were apparent in two of five patients examined 6 months after resection, six of 12 at 12 months, and three of 11 at 24 months, and were therefore not related merely to a short postoperative time interval. In a number of cases showing inflammation at the anastomosis there was also evidence of ulceration (Fig. 2) or even the formation of exuberant granulation tissue (Fig. 3). Occasionally, the formation of granulation tissue was obviously related to the presence of suture material; taken

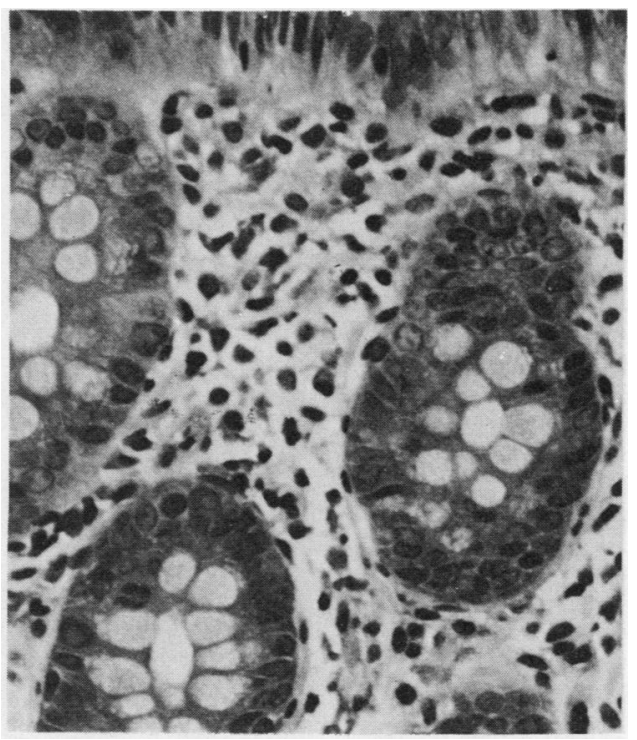

Fig. 1 Mucosa from an anastomosis site 12 months after colonic resection showing minimal inflammatory change evidenced by a slight increase in the numbers of mononuclear cells present in the lamina propria. Haematoxylin and eosin. $\times 300$.

overall there was evidence of mucosal ulceration at the anastomosis in two of five patients seen at 6 months, three of 12 at 12 months, and two of 11 at 24 months. Those cases with more florid inflammatory changes corresponded with those which had

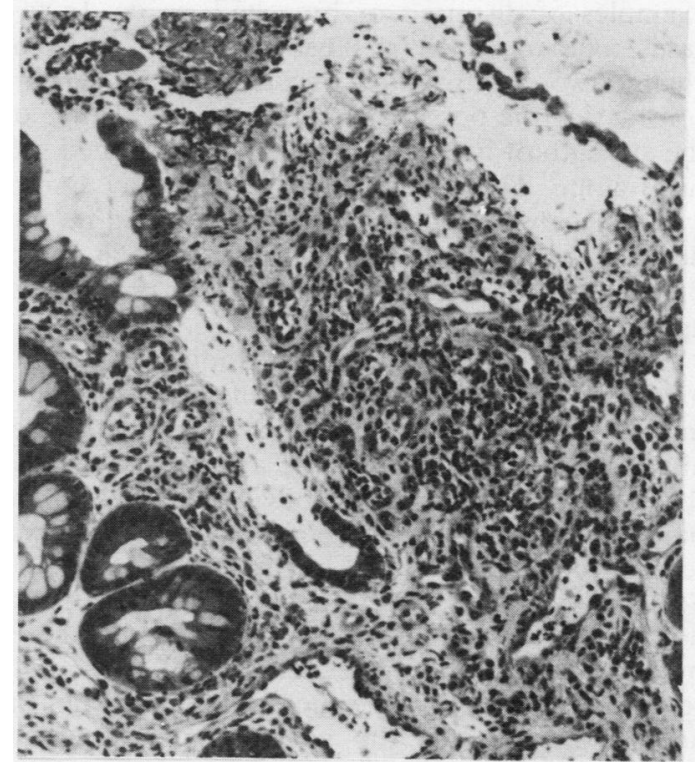

Fig. 2 Mucosa from an anastomosis 12 months after resection showing active inflammatory change including superficial mucosal ulceration. Haematoxylin and eosin. $\times 150$. 
anastomosis at 24 months after resection. Haematoxylin and eosin. $\times 150$.

been abnormal endoscopically, and both right and left sided anastomoses were affected.

In several of those cases in which inflammatory changes were apparent there were accompanying disorders of crypt architecture. These abnormalities included areas of crypt elongation or dilatation and examples of distorted or branched crypts. In two cases, one of which is illustrated (Fig. 4), some changes were sufficiently conspicuous as to warrant considering the possibility of transitional change on the strength of haematoxylin and eosin stained sections alone. There was no evidence in any case of even mild dysplastic change, however, and no evidence of recurrent or de novo carcinoma in any of the samples. No fibrosis of the lamina propria was apparent.

Staining by the diastase-periodic acid Schiff method made clear the presence of so called melanosis coli in most cases, but the extent of this abnormality was similar in the anastomoses and in the bowel as a whole. No obvious change in the pattern of mucin production at the anastomosis was seen in the section stained by diastase-periodic acid Schiff and Shikata's orcein method. Changes were, however, often demonstrable in the sections stained by the high iron diamine/alcian blue method. In seven cases one or more mucosal samples from the anastomosis showed the overwhelming predominance of sialomucins characteristic of the transitional mucosa. And in other samples a mixed pattern of mucin production was apparent, the picture being neither that of normal left colon nor that of

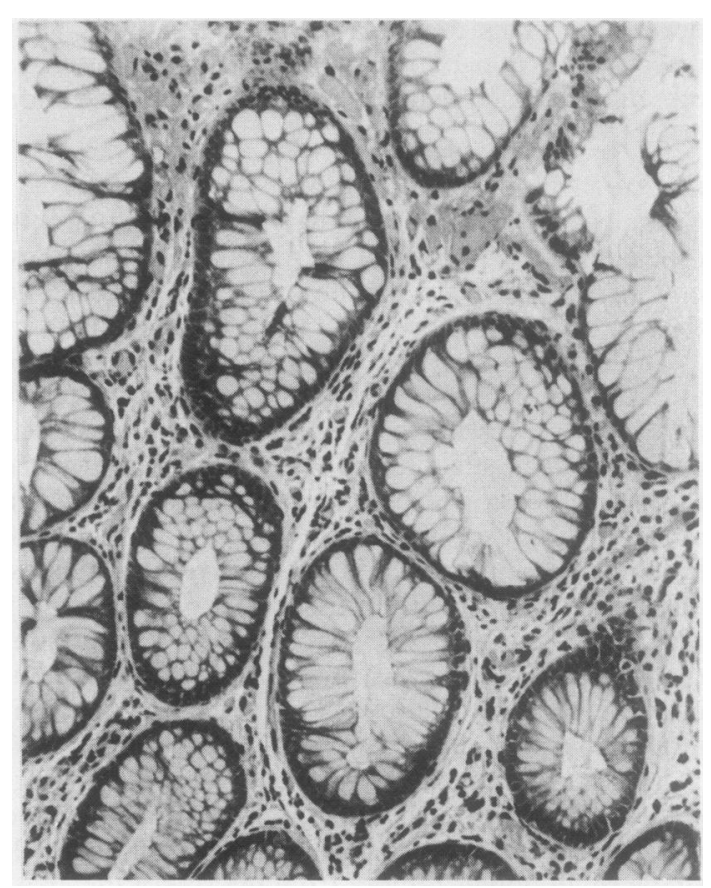

Fig. 4 Abnormal mucosa of transitional pattern present at an anastomosis 12 months after resection. Haematoxylin and eosin. $\times 150$.

normal right colon. This mixed pattern of mucin production was seen elsewhere in the bowel in nine patients, and it is thus difficult to regard it as particularly important, but transitional change did appear to be confined to the site of the anastomosis. In order to exclude the possibility that change may have been present at the time of the original surgical resection-that is, that it was not acquired subsequent to anastomosis - sections from the line of resection blocks from the original surgical resections were stained by methods for mucin. Transitional 0 change was not apparent in this material.

The intensity of epithelial carcinoembryonic antigen positivity varied from case to case, but no consistent change in the pattern of expression at the anastomosis site was discerned.

\section{Discussion}

For the reasons outlined previously it is quite possible that suture line tumours represent metachronous primary growths, but this hypothesis is difficult $\stackrel{D}{~}$ to prove. In view of the obvious difficulties in interpreting the changes apparent in established suture line malignancies this study was designed to investigate whether any preneoplastic alteration in the crypt cells could be detected at the site of healthy anastomoses. It is now generally accepted that malignancies of the large bowel arise as a result of a 8 progression from various abnormalities described as 
dysplasias, both in the case of ordinary large bowel cance ${ }^{13}$ and in the setting of carcinomas complicating inflammatory bowel disease. ${ }^{15}$ But dysplastic changes were not seen at the anastomoses, and it would thus appear that fully acceptable evidence of the earliest changes of de novo tumorigenesis is lacking. Other changes at the anastomosis site were common, however, and, given the small numbers of patients included in this study and the relatively short follow up period, these may well be of importance in indicating the possibility of an enhanced risk of tumour development at the anastomosis.

Perhaps the most striking of these changes was the emergence at the anastomosis in seven cases of patchy transitional change, with a switch from a dominance of sulphomucin production within the crypt to almost entirely sialomucin production. This change has long been recognised in the vicinity of primary colonic carcinomas both in $\operatorname{man}^{16}$ and in experimental colonic cancers in rats ${ }^{17}$ and its importance as a preneoplastic change has been suggested. ${ }^{18}$ While transitional change has recently been described in situations not obviously related to the development of cancer and its significance as a preneoplastic phenomenon has been questioned, ${ }^{14} 20$ debate continues. ${ }^{21}$ It may well be that in the present material transitional change is merely a regenerative effect and that this is what accounts for its localisation to the anastomosis; but the alternative interpretation is still possible. Those cases in which it was manifest were not conspicuously those with adenomas, which is another index or risk of metachronous cancer development; ${ }^{22}$ nor was its presence related to Dukes' stage of differentiation of the original tumour.

Inflammatory changes at the anastomosis were seen in 11 cases, often with associated ulceration, and these changes were apparent up to 24 months after resection, indicating a degree of chronicity. Since it is generally accepted that any stimulus to continued cell proliferation can act as a promoting influence in the process of neoplastic development, this inflammation could certainly act to localise the development of a metachronous tumour to the anastomosis. The precise cause of the inflammation and ulceration is not clear, but it may well be related to the presence of sutures.

In conclusion, we have found no definite evidence of precancerous change at the sites of large intestinal anastomoses; there are, however, often quite striking morphological abnormalities which suggest that the anastomosis is especially vulnerable to de novo tumorigenesis. More intensive study is required, using greater numbers of patients, and perhaps especially looking at anastomoses which have been present for several years before we can be sure that large bowel anastomosis in patients with cancer does not in itself present a significant cancer risk.

We thank the technical staff of the Histopathology Laboratory, Queen Elizabeth Hospital, for their expert assistance; Mrs A Hogarth and Miss D Scott for typing successive versions of the manuscript; and Mr S Brabazon for preparing the illustrations.

References
' Slaney G. Results of treatment of carcinoma of the colon and rectum. In: Irvine W, ed. Modern trends in surgery 3. Sevenoaks, Kent: Butterworths, 1971:69-89.

${ }^{2}$ Phillips RKS, Hittinger R, Blesovsky L, Fry JS, Fielding LP. Local recurrence following 'curative' surgery for large bowel cancer: $I$. The overall picture. Br J Surg 1984;71:12-6.

${ }^{3}$ Heald RJ, Bussey HJR. Clinical experience at St Mark's Hospital with multiple synchronous cancers of the colon and rectum. Dis Colon Rectum 1975; 18:6-18.

- Goligher JC. Surgery of the anus, rectum and colon. 4th ed. London: Balliere Tindall, 1980:431

s Shamsuddin AKM, Weiss L, Phelps PC, Trump BF. Colon epithelium. IV. Human colon carcinogenesis. Changes in human colon adjacent to and remote from carcinoma of the colon. JNCI 1981;66:413-9.

- Preumont AM, Stoffels GL, de Reuck M. Nuclear size and nuclear binding of tritiated actinomycin $D$ into epithelial cells of colon cancer patients with apparently normal colorectal mucosa. Can Res 1981;41:2529-33.

' Deschner EE. Early proliferative changes in gastrointestinal neoplasia. Am J Gastroenterol 1982;77:207-11.

" Phillips RKS, Hittinger R, Blesovsky L, Fry JS, Fielding LP. Local recurrence following 'curative' surgery for large bowel cancer. II. The rectum and rectosigmoid. Br J Surg 1984;71:17-20.

- Williamson RCN, Bauer FLR, Terpstra OT, Ross JS, Malt RA. Contrasting effects of subtotal enteric bypass, enterectomy and colectomy on azoxymethane-induced intestinal carcinogenesis. Can Res 1980;40.538-43.

${ }^{10}$ Senior PV. Cell proliferation in hyperplastic and preneoplastic states in the rodent intestine. Newcastle: The University of Newcastle upon Tyne, 1983. PhD Thesis.

"Winkler R, Pfeiffer M, Ayisi K, Dömer A. Spontaneous colostomy cancer in rat: a handy model of colonic carcinogenesis. In: Malt RA, Williamson RCN, eds. Colonic carcinogenesis. Lancaster: MTP Press Limited, 1982:245-9.

12 Williamson RCN, Davies PW, Bristol JB, Wells M. Intestinal adaptation and experimental carcinogenesis after partial colectomy. Increased tumour yields are confined to the anastomosis. Gut 1982;23:316-25.

13 Day DW, Morson BC. In: Morson BC, ed. The pathogenesis of colorectal cancer. New York: WB Saunders Co, 1978:58-71.

i4 Sipponen P. Histochemical reactions of gastrointestinal mucosubstances with orcein, high iron diamine and alcian blue, after prior oxidation of tissue sections. Histochemistry 1979;59:199-206.

is Riddell RH, Showe DC, Ritchie JK, Lennard-Jones JE, Morson BC. In: Morson BC, ed. The pathogenesis of colorectal cancer. New York: WB Saunders Co, 1978:95-118.

${ }^{16}$ Filipe MI. The value of a study of the mucosubstances in rectal biopsies from patients with carcinoma of the rectum and lower sigmoid in the diagnosis of premalignant mucosa. J Clin Pathol 1972;25:123-8.

${ }^{17}$ Filipe MI. Mucous secretion of rat colonic mucosa during carcinogenesis induced by dimethylhydrazine: a morphological and histochemical study. Br J Cancer 1975;32:60-77.

18 Filipe MI, Cooke KB. Changes in composition of mucin in the mucosa adjacent to carcinoma of the colon as compared with the normal: a biochemical investigation. J Clin Pathol 1974;27:315-8.

14 Isaacson P, Attwood PRA. Failure to demonstrate specificity of the morphological and histochemical changes in mucosa adjacent to colonic carcinoma (transitional mucosa). J Clin Pathol 1979;32:214-8.

${ }^{20}$ Franzin G, Grigioni WF, Dina R, Scarpa A, Zamboni G. Mucin secretion and morphological changes of the mucosa in non-neoplastic diseases of the colon. Histopathology 1983;7:707-18.

21 Filipe MI, Transitional mucosa (letter). Histopathology 1984;8:707.

22 Bussey HJR, Wallach MH, Morson BC. Metachronous carcinoma of the large intestine and intestinal polyps. Proc R Soc Med 1967;60:208-18.
lonsey

Requests for reprints to: Dr JP Sunter, Department of Pathology, Queen Elizabeth Hospital, Sheriff Hill, Gateshead, Tyne and Wear NE9 6SX, England. 